\title{
Very slow propagating mode at audiofrequencies in gel
}

\author{
J. C. Bacri, J. Dumas and A. Levelut \\ Laboratoire d'Ultrasons (*), Université Pierre-et-Marie-Curie, Tour 13, 4, place Jussieu, 75230 Paris Cedex 05, France
}

(Reçu le 8 mars 1979, révisé le 5 avril 1979, accepté le 6 avril 1979)

\begin{abstract}
Résumé. - Nous avons mis en évidence un mode élastique (longitudinal) de gonflement à très basse fréquence (de $100 \mathrm{~Hz}$ à $2000 \mathrm{~Hz}$ ) dans un gel polyacrylamide à $10 \%$. Nous avons trouvé une vitesse de phase très faible $(2,3 \mathrm{~m} / \mathrm{s})$ directement reliée à la constante de cisaillement du réseau. Nous mesurons une viscosité liée au polymère.
\end{abstract}

\begin{abstract}
An elastic dilatational mode at very low frequency $(100 \mathrm{~Hz}$ to $2000 \mathrm{~Hz})$ has been found in a $10 \%$ polyacrylamide gel. The phase velocity, which is directly related to the polymer shear constant, is very low $(2.3 \mathrm{~m} / \mathrm{s})$. A polymer network viscosity has also been measured.
\end{abstract}

1. Introduction. - A gel is an infinite crosslinked polymer network imbedded in a fluid solvent. Mechanical vibrations in gel can be studied in three regions of frequencies which give different kinds of information :

- at high frequencies $(\gtrsim 100 \mathrm{MHz})$, the elastic constant measured is that of water slightly modified by the polymer, and the sound attenuation is related to the friction between the network and the solvent [1] ;

- at intermediate frequencies $(\simeq 1-20 \mathrm{MHz})$ the relaxations of internal motions in monomers are the main parameters affecting ultrasonic measurements [2] ;

- at very low frequencies $(0-10 \mathrm{kHz})$, static measurements and light scattering experiments $[3,4,5]$ give very low values of the elastic constant of the gel, $10^{4}$ times smaller than high frequency values.

The aim of this letter is to present direct dynamic measurements of the elastic shear constant in this very low frequency range, and some information about the viscosity of the polymer network. These results are obtained by the observation of an axially symmetrical dilatational mode in a gel cylinder.

2. Theory. - In order to interpret the present very low frequency experiment we consider the gel as a single-component solid. Our reasons for adopting this description rather than the two component model used in interpreting high frequency experiments [1]

(*) Associé au Centre National de la Recherche Scientifique. are the following. In the two-component model the displacement $\mathbf{r}$ of the polymer network and the displacement $\mathbf{s}$ of the solvent are coupled through a friction term $f(\dot{\mathbf{r}}-\dot{\mathbf{s}})[1,6]$. This term (which varies as $\omega$ ) is dominant at low frequencies as compared to the inertial terms and to the restoring force terms (which vary as $\omega^{2}$ ). As a consequence, in our frequency range $(v<2000 \mathrm{~Hz})$, the movements of the two components are in phase and they may be accurately described by a single variable. In fact the value of the friction coefficient $f \simeq 10^{10}$ dynes $\mathrm{s} . \mathrm{cm}^{-4}$ corresponds to a swelling time of the order of some days [7] : the solvent does not flow out of the polymer mesh during the duration of the experiment. Therefore, we suppose the network and solvent movements are in phase in the gel which may be considered as a single homogeneous isotropic solid. Under these conditions we have to study a sample mainly composed of a solvent held in the shape of a rod by the polymer network. This sample is a solid : it has a shear modulus which is due to the polymer. Then we may put

$$
\mu_{\mathrm{g}} \simeq \mu_{\mathrm{p}}
$$

where $\mu_{\mathrm{p}}$ is the shear modulus of the polymer network. Static measurements give an order of magnitude for this coefficient : $\mu_{\mathrm{p}} \simeq 2 \times 10^{5}$ dynes $\mathrm{cm}^{-2}$ for a $10 \%$ acrylamide gel [3]. This solid sample has a compressibility or a bulk modulus $K_{\mathrm{g}}$ which may be measured, in principle, in a hydrostatic compression measurement. The compressibility is mainly due to the solvent

$$
K_{\mathrm{g}} \simeq K_{\mathrm{s}}
$$


where $K_{\mathrm{s}}$ is the bulk modulus of the solvent. Typically, one has

$$
K_{\mathrm{s}} \simeq 2 \times 10^{10} \text { dynes } \mathrm{cm}^{-2} .
$$

In order to describe the elastic properties of an isotropic solid, two other constants may be used : the Young's modulus $E$ and the Poisson's ratio $\sigma$. They are defined as follows [8] :

$$
E_{\mathrm{g}}=\frac{9 K_{\mathrm{g}} \mu_{\mathrm{g}}}{3 K_{\mathrm{g}}+\mu_{\mathrm{g}}}, \quad \sigma_{\mathrm{g}}=\frac{1}{2} \frac{3 K_{\mathrm{g}}-2 \mu_{\mathrm{g}}}{3 K_{\mathrm{g}}+\mu_{\mathrm{g}}} .
$$

In the case of our gel, $K_{\mathrm{g}} \gg \mu_{\mathrm{g}}$. As a consequence, we have

$$
E_{\mathrm{g}} \simeq 3 \mu_{\mathrm{g}}, \quad \sigma_{\mathrm{g}} \simeq \frac{1}{2}
$$

Finally the density $\rho_{\mathrm{g}}$ is given by

$$
\rho_{\mathrm{g}}=(1-c) \rho_{\mathrm{s}}+c \rho_{\mathrm{p}}
$$

where $c$ is the polymer concentration. We have $\rho_{\mathrm{g}} \simeq 1 \mathrm{~g} / \mathrm{cm}^{3}$.

2.1 We deal first with longitudinal elastic modes in a solid rod. Its diameter $2 R$ is very small compared with $\lambda$, the mode wavelength. The cylinder axis is along $z$. In this case the only non zero components of the stress and strain tensors [8] are $\sigma_{z z}$ and

$$
u_{z z}=\frac{\partial u_{z}}{\partial z}
$$

they are related by

$$
\sigma_{z z}=C \frac{\partial u_{z}}{\partial z}
$$

where $C$ is an elastic constant which has to be defined in each experimental case. Substituting in the general dynamical equation

$$
\rho \ddot{u}_{z}=\frac{\partial \sigma_{z k}}{\partial x_{k}}
$$

we obtain for the displacement $u_{z}$ the equation

$$
\frac{\partial^{2} u_{z}}{\partial z^{2}}-\frac{\rho}{C} \frac{\partial^{2} u_{z}}{\partial t^{2}}=0
$$

which is the usual form of the propagation equation of waves with phase velocity :

$$
v=\sqrt{\frac{C}{\rho}} .
$$

1. - If the outer surface of the rod is tighly clamped, the value of $C$ which occurs is [8]

$$
C_{\infty}=\frac{E(1-\sigma)}{(1+\sigma)(1-2 \sigma)}=\frac{3 K+4 \mu}{3} .
$$

In the case of gel, $K \gg \mu$ and the mode velocity obtained is

$$
v=\sqrt{\frac{K}{\rho}} .
$$

This is the normal velocity of longitudinal waves in the solvent, in the case of infinite transverse dimensions. Numerically, $v_{\infty} \simeq 1500 \mathrm{~m} \mathrm{~s}^{-1}$ in water.

The mode observed mainly reflects properties of the solvent and the effect of the polymer network on this mode is small and can only be observed with sensitive methods [1].

2. - If the outer surface of the rod is free, the relevant elastic constant is the Young's modulus $E_{\mathrm{g}}[8]$ :

$$
C_{0}=E_{\mathrm{g}}
$$

and the phase velocity of this particular mode is

$$
v_{0}=\sqrt{\frac{E_{\mathrm{g}}}{\rho}} .
$$

For the gels, where $K \gg \mu$, we have $E_{\mathrm{g}} \simeq 3 \mu_{\mathrm{p}}$ and the velocity of this longitudinal mode depends only on the shear modulus of the polymer; thus we may predict that the velocity $v_{0}$ is slow. The Young's modulus is simply related to the shear modulus $\mu$. In these two cases (clamped and free rods) the elastic modes are non dispersive.

2.2 The discussion has been made for the condition $R \ll \lambda$, where the transverse uniformity was justified. In other cases, the two displacement components $u_{z}$ and $u_{r}$ must be considered and the calculation is complicated. This calculation has been made in numerous papers $[9,10,11]$ and we mention only the main results here. When the ratio $R / \lambda$ increases, the phase velocity $v_{\varphi}$ of the mode in the free rod decreases from the value $v_{0}$ and asymptotically tends to a constant value for $R / \lambda \rightarrow \infty$ (Fig. $1 a$ ). This value $v_{R}$ corresponds to a mode propagating in a medium with a free limiting surface which has an infinite radius

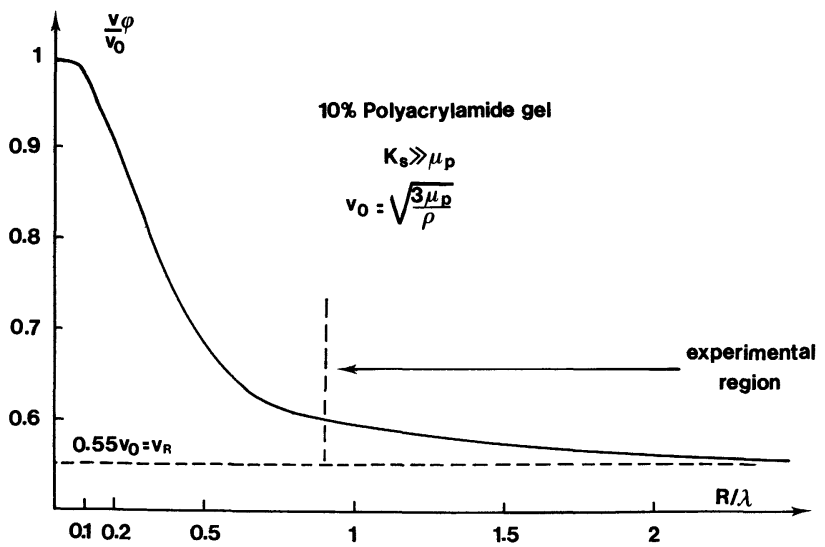

Fig. 1. - a) Theoretical dispersion curve for a dilatational mode. The phase velocity is plotted against $R / \lambda . R=\operatorname{rod}$ radius, $\lambda=$ mode wavelength (from references $[9,10]$ ). 


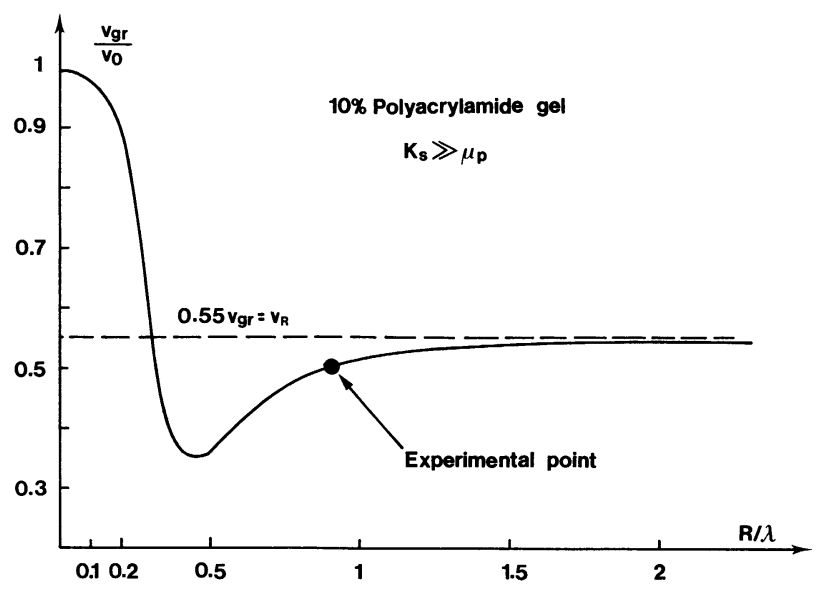

Fig. 1. - $b$ ) Group velocity for a dilatational mode plotted against $R / \lambda$. $R=$ rod radius, $\lambda=$ mode wavelength (from reference [11]).

of curvature; it is a Rayleigh wave in a plane. In the case of interest for us (where $\sigma \simeq \frac{1}{2}$ ) it is found that $[8,10]$ :

$$
v_{R} \simeq 0.55 v_{0}=0.55 \sqrt{3} \sqrt{\frac{\mu}{\rho}}=0.96 \sqrt{\frac{\mu}{\rho}} .
$$

The schematic variation of $v_{\varphi}(R / \lambda)$ is given in figure $1 a$. The behaviour of the group velocity $v_{\mathrm{gr}}(R / \lambda)$ of this mode is more complicated. Starting from $v_{\mathrm{gr}}=v_{0}$ for $R=0$, it decreases, passes through a minimum (typically $0.4 v_{0}$ ) for $R / \lambda \sim 0.5$ and tends towards the value $v_{R}$ for $R / \lambda \rightarrow \infty$. This variation is also reproduced in figure $1 b$.

2.3 We have not yet mentioned viscosity effects in the previous discussion. To include them, it is sufficient to remark that the bulk viscosity $\xi$ and the shear viscosity $\eta$ which appear in the treatment of isotropic media $[8,12]$ may be taken into account by means of imaginary parts of the bulk modulus $K$ and the shear modulus $\mu$, giving the complex. elastic moduli :

$$
\begin{aligned}
& \bar{K}=K+i \omega \xi \\
& \bar{\mu}=\mu+i \omega \eta .
\end{aligned}
$$

The complex coefficients which are of interest for us are :

$$
\bar{E}=\frac{9 \bar{K} \bar{\mu}}{3 \bar{K}+\bar{\mu}}, \quad \bar{\sigma}=\frac{1}{2} \frac{3 \bar{K}-2 \bar{\mu}}{3 \bar{K}+\bar{\mu}} .
$$

In the particular case where $K \gg \mu$ we obtain

$$
\bar{E} \simeq 3(\mu+i \omega \eta), \quad \bar{\sigma} \simeq \frac{1}{2}-i \frac{\omega \eta}{2 K} .
$$

Then, for our gel, we have :

$$
\bar{E} \simeq 3 \mu_{\mathrm{g}}+i \omega 3 \eta_{\mathrm{g}}, \quad \bar{\sigma} \simeq \frac{1}{2}
$$

where $\eta_{\mathrm{g}}$ is the shear viscosity of the whole gel. Therefore, the acoustic losses in a thin $\operatorname{rod}(R / \lambda \ll 1)$ are governed by an effective viscosity coefficient $\eta_{0}=3 \eta_{\mathrm{g}}$. In the opposite limit of a Rayleigh wave $(R / \lambda \gg 1)$ the complex wave vector $\bar{q}$ and the complex shear modulus are related by [8] :

$$
\omega=0.96 \sqrt{\frac{\bar{\mu}}{\rho}} \bar{q} \text { when } \quad \sigma=\frac{1}{2} .
$$

The imaginary part of $\bar{q}$ is easily deduced :

$$
q^{\prime \prime}=\frac{(0.96)^{2} \omega^{2} \eta}{2 \rho v_{R}^{3}}
$$

The losses are given by an effective viscosity coefficient $\eta_{R}=0.92 \eta$.

Our propagation experiment is done in conditions where $v_{\varphi} \simeq v_{R}$ and we assume that the previous result is relevant then; therefore we measure $\eta^{\prime} \simeq 0.9 \eta_{\mathrm{g}}$

3. Experimental. - The material used is a polyacrylamide gel in water. The polymer concentration is $10 \%$ in weight. Two samples were used in our experiments : a cylindrical rod $(45 \mathrm{~mm}$ length, $16 \mathrm{~mm}$ in diameter) and a circular disc $(3.5 \mathrm{~mm}$ high, $16 \mathrm{~mm}$ in diameter). We have made two successive measurements : one with pulsed waves on the rod, and one with continuous waves on the disc.

3.1 SAMPLE PREPARATION. - The polyacrylamide gel was prepared in the following way, $30 \mathrm{~g}$ acrylamide and $0.8 \mathrm{~g} \mathrm{~N}, \mathrm{~N}^{\prime}$-methylene-bis-acrylamide (a crosslinking agent) were dissolved in distilled water to make up a $100 \mathrm{ml}$ solution (solution A). $3.33 \mathrm{ml}$ of this solution A was transferred into a test-tube ; $330 \mu \mathrm{l}$ of $10 \%$ ammonium persulfate solution was added with distilled water to make up a $10 \mathrm{ml}$ solution. $5 \mu \mathrm{l}$ of tetraethyl-N-ethylene-diamine was further added to initiate the copolymerization of the acrylamide and bis-acrylamide solution. The gelation occurred 10 minutes later. The problem is to remove the gel from the test tube without damage. This is done by carefully introducing water between the gel and the tube wall with a syringe. The sample is cut to the desired size with a scalpel and a thin nylon wire.

3. 2 Pulsed Waves IN THE ROD. - A $260 \mathrm{~Hz}$ pulse of current of $10 \mathrm{~ms}$ duration is applied to an electromechanical transducer which gives a longitudinal displacement to the entire end surface of the gel rod. A second transducer in contact with the opposite face acts as a receiver. The outer surface of the rod is kept perfectly free. The transit time between different echoes gives the group velocity of the gel mode excited at this frequency. We made the measurements on three pulses and we find

$$
v_{\mathrm{gr}}=(220 \pm 30) \mathrm{cm} / \mathrm{s} .
$$


This very low value shows that the acoustic impedance mismatch between transducer and gel is high and therefore the reflection is quasi total. The wave velocity in gel is about $10^{2}$ lower than in the surrounding air : the elastic energy cannot be radiated because of total reflection. Moreover, the losses due to viscoelastic wave emission in air are negligibly small. Scattering from sample inhomogeneities is also not a relevant cause of attenuation considering the wavelength of the waves $(0.1 \mathrm{~cm}$ to $1 \mathrm{~cm})$ and the excellent optical quality of the sample on this scale.

Thus the exponentially decreasing echo amplitude $A_{n}$ ( $n$ : transmitted echo number) gives a measurement of the true attenuation of the mode

$$
\frac{A_{n+1}}{A_{n}} \propto \mathrm{e}^{-q^{\prime \prime l}}
$$

where $l$ is the sample length. Figure 2 shows the exponential envelope of the transmitted echoes which corresponds to an attenuation

$$
q^{\prime \prime} \simeq 2 \times 10^{-6} v^{2} \mathrm{~cm}^{-1}
$$

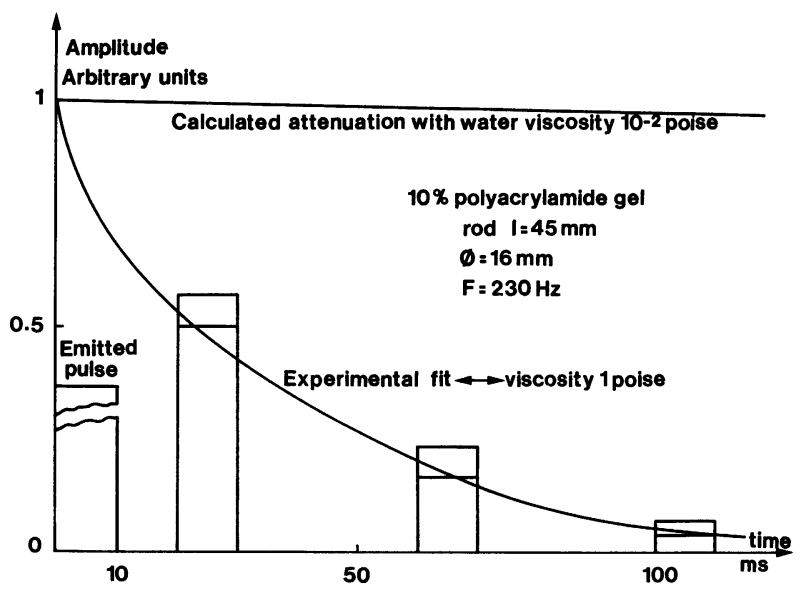

Fig. 2. - Measurement of transit time and attenuation of the dilatational mode for transmitted pulses. - Experimental fitting corresponding to a $1 \mathrm{P}$ viscosity ; - Calculated curve for attenuation in the same length of water, viscosity $\eta=0.01 \mathrm{P}$.

where the frequency $v=\omega / 2 \pi$ is given in $\mathrm{Hz}$. This corresponds to a measured viscosity :

$$
\eta^{\prime} \simeq \eta_{\mathrm{g}} \simeq 1 \mathrm{P}
$$

$\eta_{\mathrm{g}}$ is then, roughly, 100 times the solvent viscosity $\left(\eta_{\text {water }} \simeq 10^{-2} \mathrm{P}\right)$.

The total viscosity is the result of several contributions :

- the viscosity of the network due to deformation of the polymer chains,

- the shear viscosity of the solvent,

- the friction term which arises from the relative movement of solvent and solute : this term can be ruled out since solvent and solute move in phase in the modes we consider.
The viscosity we measure seems very likely to be, under these conditions, the viscosity of the polymer network :

$$
\eta_{\mathrm{p}} \simeq 1 \mathrm{P}
$$

The experimental conditions of measurement on three pulses show that this value should be taken only as an order of magnitude.

3.3 Continuous waves IN THE DISC. - The disc of gel is maintained directly between the same two transducers. The lateral surface is kept free. The emitting transducer is driven by a continuous wave and the transmitted amplitude of sound is measured as a function of frequency : maxima in the signal are observed for sound frequencies for which the sample has a length of $n \frac{\lambda}{2}, n=1,2,3, \ldots$ Figure 3 gives

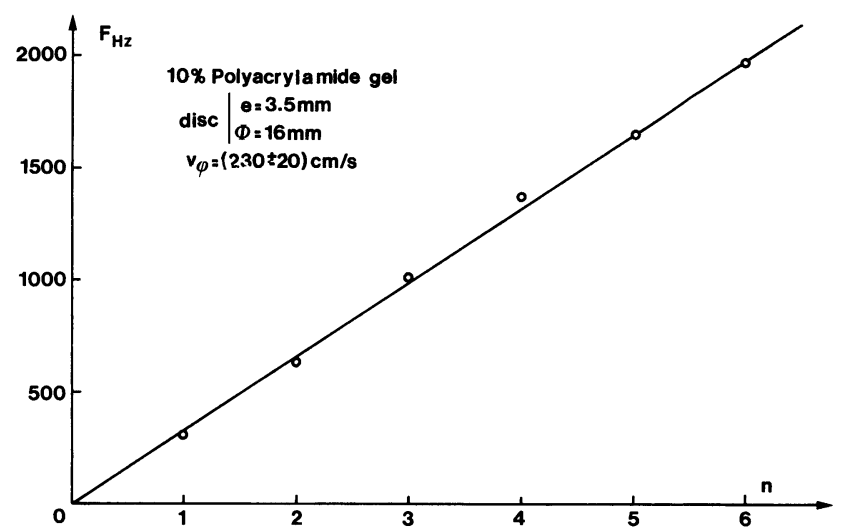

Fig. 3. - Experimental dispersion curve of the dilatational mode obtained from $\mathrm{CW}$ method in $10 \%$ polyacrylamide gel. The resonance frequencies of the sample are plotted against the order of excited harmonics. The first harmonic corresponds to a wavelength about $7 \mathrm{~mm}$.

the resonance frequency as a function of $n$. It is possible to deduce a phase velocity

$$
v_{\varphi}=(230 \pm 20) \mathrm{cm} / \mathrm{s}
$$

which shows no dispersion within our experimental accuracy.

4. Discussion. - The phase velocity measurements are made between 200 and $2000 \mathrm{~Hz}$; the corresponding wavelengths are between 1 and $0.1 \mathrm{~cm}$ and therefore the ratio $R / \lambda$ varies between 0.8 and 8 . In this region the expected variations of $v_{\varphi}$ are less than $5 \%$ (see $[9,10]$ and our figure $1 a)$. Our experimental results which give a dispersionless velocity with an accuracy of $10 \%$ are in agreement with the theory.

For our group velocity measurement made with $R / \lambda \simeq 0.8$, it is expected that $v_{\mathrm{gr}} \simeq 0.85 v_{R} \simeq 0.85 v_{\varphi}$. Our experimental result (which is less accurate than for $v_{\varphi}$ ) is compatible with the theoretical prediction. Taking

$$
v_{\varphi}=0.55 \sqrt{\frac{3 \mu_{\mathrm{p}}}{\rho}}=230 \mathrm{~cm} / \mathrm{s} \text {, }
$$


Table I. - Comparison between the different elastic coefficients of the solvent, the polymer network and the whole gel.

\begin{tabular}{|c|c|c|c|c|c|}
\hline & $\begin{array}{l}\text { Compressibility } \\
\text { modulus }\end{array}$ & $\begin{array}{c}\text { Young } \\
\text { modulus }\end{array}$ & $\begin{array}{l}\text { Poisson } \\
\text { ratio }\end{array}$ & $\begin{array}{c}\text { Shear } \\
\text { modulus }\end{array}$ & $\begin{array}{c}\text { Longitudinal } \\
\text { elastic constant } \\
C\end{array}$ \\
\hline & $K$ & $E$ & $\sigma$ & $\mu$ & $C_{11}$ \\
\hline $\begin{array}{l}\text { Solvent } \\
\text { Polymer } \\
\text { Gel }\end{array}$ & $\begin{array}{c}K_{\mathrm{s}} \\
\frac{2}{3} \mu_{\mathrm{p}} \\
K_{\mathrm{s}}+\frac{2}{3} \mu_{\mathrm{p}} \simeq K_{\mathrm{s}}\end{array}$ & $\begin{array}{c}0 \\
2 \mu_{\mathrm{p}} \\
3 \mu_{\mathrm{p}}\left({ }^{b}\right)\end{array}$ & $\begin{aligned} & \frac{1}{2} \\
& 0 \\
\simeq & \frac{1}{2}\end{aligned}$ & $\begin{array}{c}0 \\
\mu_{\mathrm{p}} \\
\mu_{\mathrm{p}}\end{array}$ & $\begin{array}{c}K_{\mathrm{s}} \\
2 \mu_{\mathrm{p}}\left({ }^{c}\right) \\
K_{\mathrm{s}}+2 \mu_{\mathrm{p}} \simeq K_{\mathrm{s}}\left({ }^{a}\right)\end{array}$ \\
\hline
\end{tabular}

$\left({ }^{a}\right)$ Measured in reference [1].

$(b)$ These measurements and static measurement $[3,13]$.

${ }^{(c)}$ Elastic constant measured in light diffusion experiment $[3,13,14]$.

we find

$$
\mu_{\mathrm{p}}=\mu_{\mathrm{g}}=(5.8 \pm 0.3) 10^{4} \text { dynes } / \mathrm{cm}^{2} .
$$

Static measurement in a $10 \%$ polyacrylamide gel [3] has given $E \simeq 20 \times 10^{4}$ dynes $/ \mathrm{cm}^{2}$. Our own measurements give

$$
E=3 \mu_{\mathrm{g}}=(17.5 \pm 1) 10^{4} \mathrm{dynes} / \mathrm{cm}^{2} .
$$

These two results are in agreement.

The dispersion curves drawn in figure $1 a$ and $b$ are relative to a mode which has no nodal cylindrical surface. Other modes exist which correspond to vibration with one or more nodal cylindrical surfaces and have different dispersion curves $[9,11]$. But a uniform excitation on the end surface is not likely to excite these modes which correspond to a very non-uniform distribution of displacements over the cross-section of the cylindrical rod [11].
In table I, we recapitulate the various elastic coefficients involved in a gel and we indicate which experimental method allows one to obtain some of them. $\mu_{\mathrm{p}}$ which is a particularly important parameter of the gel, has been measured in our experiment. It may also be obtained by light scattering experiments $[3,13,14]$.

5. Conclusion. - We have reported on a simple experimental method which gives the shear modulus $\mu_{\mathrm{p}}$ and the polymer network viscosity of a gel.

Other methods could give the same type of information. For instance, transverse bulk elastic wave propagation may provide values of $\mu_{\mathrm{p}}$ and $\eta_{\mathrm{p}}$ but it is more complicated to achieve. On the other hand, static experiments measure $\mu_{\mathrm{p}}$ but are unable to give the viscosity. It seems that our technique has some advantages over the above methods.

\section{References}

[1] BaCRi, J.-C., Rajaonarison, R., J. Physique Lett. 40 (1979) L-5.

[2] Pugh, D., Jones, D. A., Polymer 19 (1978) 1008.

[3] Hecht, A. M., Geissler, E., J. Physique 39 (1978) 631.

[4] Brenner, S. L., Gelman, R. A., Nossal, R., Macromolécules 11 (1978) 202.

[5] Nossal, R., Brenner, S. L., Macromolécules 11 (1978) 207.

[6] De Gennes, P. G., Macromolécules 9 (1976) 587.

[7] Tanaka, T., Ishiwata, S., Ishimoto, C., Phys. Rev. Lett. 38 (1977) 771.

[8] Landau, L., Lifchitz, E., Théorie de l'Elasticité (Ed. MIR, Moscou).
[9] Redwood, M.-R., Mechanical waveguides (Pergamon Press) 1960, Chap. 6.

[10] BanCRoFt, D., Phys. Rev. 59 (1941) 588.

[11] Davies, R. M., Phil. Trans. R. Soc. A 240 (1948).

[12] Landau, L., Lifchitz, E., Mécanique des Fluides (Editions MIR, Moscou)

[13] Tanaka, T., Hocker, L. O., Benedek, G. B., J. Chem. Phys. 59 (1973) 5151

[14] Munch, J. P., Candau, S., Duplessix, R., Picot, C., Benort, H., J. Physique Lett. 35 (1974) L-239. 